\title{
Marrella: A Tool for Simulation and Verification
}

\author{
Dominique AMBROISE \\ Universite de Caen et URA 1526 CNRS \\ G.R.E.Y.C. --Esplanade de la Paix, 14032 Caen cedex \\ e_mail Dominique.Ambroise@info.unicaen.ft
}

\author{
Brigitte ROZOY \\ Université de Paris XI et URA 410 CNRS $^{\text {l }}$ \\ L.R.I., Bât. 490,91405 Orsay cedex, France \\ Tel (33 1) 69416609 , e_mail rozoy@ri.fr
}

\begin{abstract}
This paper presents the structure of our tool Marrella the construction of which has been motivated by practical problems in the context of simulation and parallel program debugging, where the correct evaluation of global properties requires a careful analysis of the causal structure of the execution. The underlying model is based on prime event structures that are considered as exhibiting all the behaviors of distributed programs : the tool gives the possibilities of generating one, some or all of their executions. On one hand, a careful implementation spares memory; on the other hand, precise and neat algorithms benefit from the trace properties of prime event structures and thus gain in avoiding the enumerations of equivalent interleavings.
\end{abstract}

\section{Introduction}

It is now well known and recognized that one execution of a distributed program may be symbolized by a partial order. In the same spirit, we claim that prime event structures can be used to exhibit in a single object all possible executions. Thus their use may become of a great help for both formal specifications, simulations and verifications of such programs. Inherently, our method is based on prime event strictures and exploits technics that unfold the behaviors of programs into acyclic nets. In fact and although not formulated in that terms, this idea has already been used for verification purposes in [MacM. 92] where unfoldings of occurrence Petri Nets are constructed. In case of distributed programs and as it partially avoids the famous state explosion problem, we think that this model is well adapted for efficient simulations, even exhaustive, thus for verifications. Substantial efficiencies are obtained as the enumeration of all possible interleaving are avoided. Therefore our tool Marrella has heavily been based on these prime event structures.

\section{The Levels in Marrella}

The description of the system is made at three distinct levels. Starting with a concrete distributed asynchronous algorithm, the tool Marrella implements an object $\Re$ that describes both the network and the algorithm. It allows to construct $G(\Re)$, the graph of states of the system, actually implemented either totally or piece by piece in case it is too big ; this graph is shown to be isomorphic to the graph of configurations of some prime event structure $\mathscr{S}: \mathscr{G}(\mathscr{R}) \approx \mathscr{C}$ onf $(\mathscr{P})$. This prime event structure $\mathscr{P}$ constitutes our abstract level, not implemented but used to derive properties of $\mathscr{Q}\left(\Re_{0}\right)$, therefore to build efficient algorithms.

This is to put side by side to the partial order associated with any distributed computation that is used to verify the associated lattice of ideals.

The real implemented object : a distributed program $\mathscr{R}$.

First of all, the really implemented object $R$ is some kind of network the nodes of which are automaton equipped with buffers and communicating by channels; this specification is very closed to an implementation using an Estelle like language [Amb. 96].

1 This work has partially been supported by the inter PRC project "Modèles et Preuves du Parallelisme" of the french MESR. 
The static description involves a network of communicating asynchronous processes together with a collection of in-coming and out-coming buffers : a set of processes and for any process $p$ a set Act(p) of firable actions, a set State(p) of (local) states, at last sets Mes(p) of messages and Buff(p) of buffers.

The dynamic part expresses the ability for any process to fire actions, to change states and to send messages. As a basic hypothesis is that the system is asynchronous and without shared memory, the resulting functions are locally testable. For every process $p$, firts the boolean function $g_{p}:$ State $(p) \times \operatorname{Buff}(p) \times \operatorname{Act}(p) \rightarrow$ Boolean allows to compute the enable actions. Second, if an action " $a$ " is possible at state " $s$ " by process " $p$ " with buffers " $\mathrm{b}$ ", then $f_{\mathrm{p}}(\mathrm{s}, \mathrm{b}, \mathrm{a})$ and $\mathrm{h}_{\mathrm{p}}(\mathrm{s}, \mathrm{b}, \mathrm{a})$ express the resulting modifications where $f_{\mathrm{p}}$ and $\mathrm{h}_{\mathrm{p}}$ are local functions, $f_{p}: \operatorname{State}(p) \times \operatorname{Buff}(p) \times \operatorname{Act}(p) \rightarrow \operatorname{State}(p) \times \operatorname{Buff}(p)$ and $h_{p}: \operatorname{State}(p)$ $\times \operatorname{Buff}(\mathrm{p}) \times \operatorname{Act}(\mathrm{p}) \rightarrow \operatorname{Buff}\left(q_{1}\right) \times \cdots \times \operatorname{Buff}\left(q_{v}\right)$.

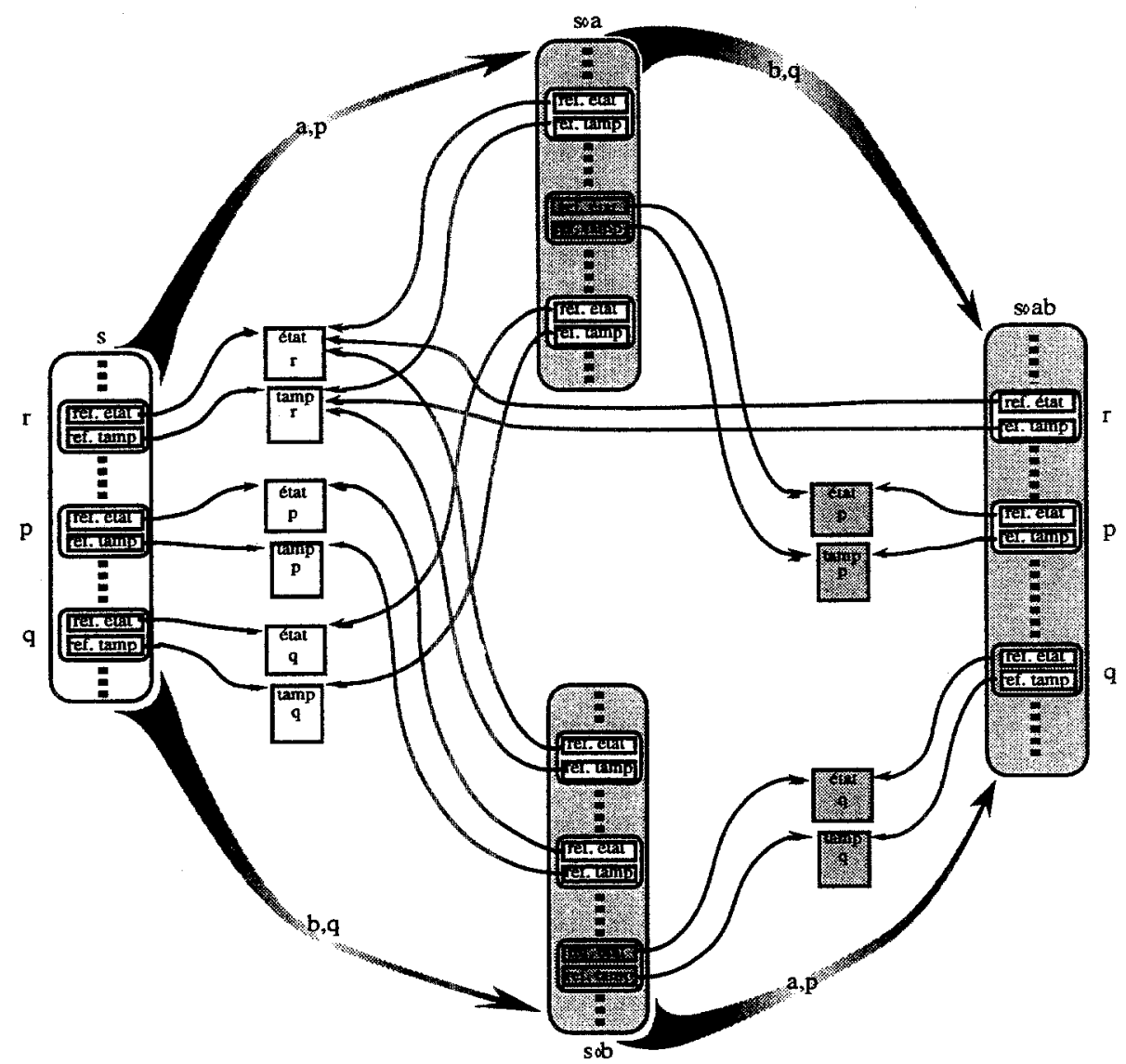

Independent computations of the modifications due to independent actions

A global state of such a system is a collection of local states $s_{k}$ together with the contents of in-coming and out-coming buffers $\mathrm{B}_{\mathrm{k}}$. Given an initial (global) state, the labeled graph of states of the system is classically defined as the graph whose nodes are the reachable 
states $\left(s_{1}, \ldots, s_{n}, B_{1}, \ldots, B_{n}\right)$, expressing the fact that every process $P_{k}$ has reach a state $s_{k}$ and buffers $B_{k}$; the arrows are labeled by the (individual) actions used to perform the step. We use another graph $\$(\Re)$, which is called the unfolded labeled graph of states of the system : if at the current stage every process $P_{k}$ has executed a sequence $w_{k}$ of actions, reaching state $s_{k}$ and buffers $B_{k}$, then the associated global unfolded state is $\left(s_{1}, \ldots, s_{n}, B_{1}\right.$, $\left.\ldots, B_{n},\left[w_{1}\right], \ldots,\left[w_{n}\right]\right)$, where $\left[w_{k}\right]$, is the trace equivalence class of the sequence of actions $\mathrm{w}_{\mathrm{n}}$. It is clear that any construction and exploration of the unfolded graph $\Theta(R)$ allows a similar approach on the graph of states.

\section{The abstract level : a prime event structure $\mathscr{\varphi}$}

At an abstract level, the description uses a labeled prime event structure that will not be constructed but is useful both to understand the properties of the graph and to derive an algorithm for constructing it. Whereas a partial order representation stands for one execution of a distributed program, an event structure exhibits in a single object all its possible executions. In that sense event structures are closed to Petri Nets with which they have strong connections [Nie. Plo. Win. 81], [Wins. Niel. 95], while prime event structures are a special case related to traces [Maz. 87].

The semantic naive interpretation of a labeled prime event structure $\mathscr{\varphi}=(\mathrm{E},<, \#, \lambda, A, \pi$, $P$ ) describes $A$ as the set of actions possible by processes $p$ in $P$ and elements of $E$ as occurrences of actions performed by these processes during the executions : $e \in E, \pi(e)=p$ and $\lambda(e)=$ a may be understood as $:$ an event $e$ will be performed by the process $p$ that fires the action a. The relation < is the classical happened before Lamport relation [Lam. 78] whereas the conflict \# may be viewed as the impossibility for two events to belong to the same behavior : at some point of the execution a choice has been made between two events and in consequence events that causally follow one of them will never follow the other.

Similarly to PoSets for which the graph is a lattice of ideals ${ }^{1}$, event structures theory gives a great place to the notion of configurations which are there an account of global states : a configuration is any past closed and conflict free subset of events. The graph of configurations is classically defined as the transition system $\mathscr{G}$ onf $(\mathscr{P})=(\mathscr{B} f(\mathscr{Y}), \mathrm{E}, \emptyset, \longrightarrow)$ where $\mathscr{C}_{f}(\mathscr{\mathscr { P }})$ is the set of finite configurations and $(\mathrm{C}, \mathrm{e}, \mathrm{C}) \in \longrightarrow$ iff $\mathrm{e} \notin \mathrm{C}$ and $\mathrm{C}^{\prime}=\mathrm{C} \cup$ $\{e\}$.

It is well known that the set of configurations of an event structure satisfies certain important domains properties and that the original structure may be recovered from its set of configurations using prime elements [Nie. Plo. Win. 81]. These properties are not far from those of lattices : these latter are prime event structures with an empty conflict relation. Here the set of finite configurations is no longer a lattice but admits however a formal characterization : it may be called a budding lattice.

The symbolic level : a budding lattice $\mathscr{G}$

The last description of the system consists in $\mathscr{Q}(\mathscr{R})$, the unfolded labeled graph of reachable states which arises to be isomorphic to the graph of configurations of a prime event structure.

\section{Theorem}

$\mathscr{G}(\Re)$, the unfolded graph of states associated to the starting real object $\mathscr{R}$ above, is isomorphic to $\mathscr{G o n f}(\mathscr{\mathscr { C }})$, the PoSet of finite configurations of some labeled event structure.

\footnotetext{
${ }^{1}$ Ideals being also called global sates or consistent cuts.
} 
Therefore, using this result, efficient constructions and traversals of this graph are implemented in our tool Marrella [Amb. Roz. 96].

\section{3 - Conclusion}

The first interest of such a construction is that Marrella uses algorithms that benefit by this structure of budding lattice : applied to prime event structures, we combine techniques that are well known on partial orders as on-the-fly model checking [Fer. Mou. Jar. Jér. 92], traces [Kat. Pel. 92], [Pel. 94] and partial order reductions [God. Wol. 91], [Val. 93], [Esp. 93]. This lead to optimal algorithms that construct either a tree th that covers the graph or the graph 8 itself ; note that, depending on the size, they may either be totally constructed or investigated piece by piece. Similarly, these properties are used to derive adequate simulation strategies [Amb. Roz. 96].

The second interest lies in the implementation : as event structures clearly exhibit the notion of event, the construction and the simulation identify events without ambiguity, thus the action of a given event is computed, executed and stored only once, what is a notable profit both in time and space. Moreover, independent actions may be executed independently on distinct parts of the memory, thus distributed simulations are conceivable.

\section{References}

[Amb. 96] D. Ambroise, Marrella ou la simulạtion guidée d'algorithmes répartis sur réseaux, thèse de l'université de Paris 11 , en préparation.

[Amb. Roz. 96] D. Ambroise, B. Rozoy, Using Event Structures for the effficient analysis of states graphs, T.R. 96, Paris-Orsay University.

[Esp. 93] J. Esparza, Model checking using net unfoldings, TapSoft'93, Orsay, LNCS $n^{\circ}$ 668, 613-628.

[Fer. Mou. Jar. Jér. 92] J.C. Fernandez, L. Mounier, C. Jard, T. Jéron, On the fly verification of finite transition systems, Formal Methods in System Design, Kluwer, 251-273.

[God. Wol. 91] P. Godefroid, P. Wolper, Using Partial Orders for the efficient verification of deadlock freedom and safety properties, CAV'91, LNCS n ${ }^{\circ} 575$, 332-342.

[Kat. Pel. 92] S. Katz, D. Peled, Verification of distributed programs using representative interleaving sequences, Distributed Computing 6, 107-120.

[Lam. 78] L. Lamport, Time, clocks and the ordering of events in a distributed system, Communications of the ACM, 21(7), 558-565, July 1978.

[MacM. 92] K. L. MacMillan, Using Unfoldings to Avoid the State Explosion Problem in the Verification of Asynchronous Circuits, CAV'92, LNCS n $^{\circ} 663$, p. 164-177.

[Maz. 87] A. Mazurkiewicz, Trace Theory, Advanced Course on Petri Nets, Bad Honnef, Germany LNCS $n^{\circ} 254,269-324$.

[Nie. Plo. Win. 81] M. Nielsen, G. Plotkin, G. Winskel, Petri Nets, Event Structures and Domains, Theor. Comp. Sci. 13 (1981) p. 85-108.

[Pel. 94] D. Peled, Combining partial order reduction with on-the-fly model checking, Proc. $6^{\text {th }}$ Int. Conf. on computer aid verification, Greece, CAV'93, 409-423.

[Val. 93] A. Valmari, On the fly verification with stubborn sets, Proc. $5^{\text {th }}$ Int. Conf. on computer aid verification, Greece, CAV'93, 397-408.

[Wins. Niel. 95] G. Winskel, M. Nielsen, Models for concurrency, in Handbook of Logic in Computer Science (S. Abramsky, DM. Gabbay, TSE. Maibaum eds.). 